\title{
El pensamiento de los futuros maestros de inglés en Educación Primaria: creencias sobre su autoeficacia docente
}

\author{
Ana Fernández-Viciana y Alberto Fernández-Costales \\ Universidad de Oviedo, España
}

(Artículo recibido el 5/10/2016; aceptado el 20/12/2016; versión final 25/02/2017)

DOI: http://dx.doi.org/10.5565/rev/jtl3.684

\begin{abstract}
Resumen
Este artículo presenta una investigación original sobre la autoeficacia docente y la formación lingüística percibida por los estudiantes del Grado en Educación Primaria (mención de inglés) de una universidad pública española. La investigación utiliza una muestra de 4 alumnos a los que se realizó una entrevista que incluía 21 preguntas. Los resultados obtenidos tras el análisis cualitativo sugieren que los participantes cuyo nivel es inferior al B2 muestran una autoeficacia lingüística baja. En líneas generales, los alumnos reclaman una mayor atención a la formación lingüística recibida en sus estudios, ya que esta resulta determinante para modelar su autoeficacia lingüística.
\end{abstract}

Palabras clave: autoeficacia, formación del profesorado, Educación Primaria, enseñanza del inglés, aprendizaje de lenguas extranjeras

\begin{abstract}
This paper reports on an original research on teacher efficacy and the perceived linguistic training of students of the Degree in Primary Education (English stream) in a Spanish university. The research relies on a sample of 4 students answered a 21-item interview. The results show students report a low language self-efficacy in the case of students with levels below B2. Students demand more attention should be paid to the learning of the L2 in their degrees and they are clearly influenced by the way their learned the foreign language at school.
\end{abstract}

Keywords: self-efficacy, teacher training, language competence, Primary Education, English teaching

\section{Résumé}

Cet article présente une recherche originale sur l'auto-efficacité sur l'enseignant auto-efficacité et la formation linguistique perçue pour les élèves de la formation des enseignants de qualité dans l'enseignement primaire (mention anglais) d'une université espagnole. La recherche utilise un échantillon de 4 étudiants qui ont réalisé un entretien avec 21 questions. Les résultats de l'étude montrent une auto-efficacité linguistique faible, notamment chez les étudiants ayant un niveau en anglais inférieur à BS. Les étudiants réclament que l'on accorde une attention spéciale à cet aspect et déclarent être nettement influencés par la manière dont ils ont appris la langue étrangère à l'école.

Mots clé: auto-efficacité, formation des enseignants, compétence linguistique, Education Primaire, enseignement de l'anglais 


\section{Introducción}

Este artículo destaca la necesidad de analizar las percepciones de los futuros docentes de inglés sobre su capacidad docente, para diseñar medidas que se orienten a remediar sus posibles carencias. Se realizó una investigación con una muestra de 4 alumnos del Grado de Maestro en Educación Primaria (mención de inglés), quienes realizaron una entrevista individual en la que respondieron a 21 preguntas (ver Anexo). Esta investigación adquiere relevancia dados los escasos estudios realizados en esta línea en España, si bien la autoeficacia docente ha sido abordada en el panorama internacional (Covarrubias \& Mendoza, 2013; de la Torre y Casanova, 2007; Mojavezi \& Tamiz, 2012; Pendergast, Garvis \& Keogh, 2011; Tschannen-Moran \& Woolfolk, 2007; Wertheim \& Leyser, 2002), resultando ser un factor esencial en la formación del profesorado. En este artículo, entendemos por creencias de autoeficacia docente la percepción de los maestros sobre su propia capacidad para llevar a cabo acciones docentes. La autoeficacia docente está relacionada con la motivación para enseñar, la toma de decisiones dentro el aula, y las estrategias metodológicas, influyendo estos factores en los resultados de los alumnos, su motivación por aprender, y sus resultados (Reoyo, 2013). Este trabajo toma como referentes estudios previos sobre creencias del profesorado (Barrios Espinosa, 2004, 2006 y 2014; Palou, 2008) y creencias de autoeficacia docente realizados en España (de la Torre y Casanova, 2007; Prieto, 2001 y 2007), aportando una contribución original al campo de la formación del profesorado al considerar las percepciones de futuros maestros de inglés sobre su competencia lingüística en el contexto de los nuevos planes de estudio adaptados al Espacio Europeo de Educación Superior (EEES). El artículo busca llenar un hueco en la literatura científica al analizar el concepto de autoeficacia docente valorando la importancia que tiene la formación lingüística que reciben los maestros.

En España, la formación de los docentes de inglés tiene una importancia superlativa debido a los resultados negativos de informes oficiales, en los que aparece regularmente por detrás de otros socios europeos en dominio de lenguas extranjeras (European Commission, 2012a; European Commission, 2012b; Eurydice, 2012).

El dominio de lenguas extranjeras en España es un problema complejo (Rubio \& Martínez Agudo, 2008) motivado por diferentes causas: primero, el español es una lengua de prestigio internacional $\mathrm{y}$, habitualmente, los ciudadanos de países con una lengua más extendida tienen un peor dominio de otros idiomas. En la Unión Europea, por ejemplo, los 3 estados miembros en los que el inglés es un idioma oficial -Reino Unido, Irlanda, y Malta- 
son los peor situados en el último eurobarómetro (European Commission, 2012a); segundo, las investigaciones en traducción audiovisual demuestran que los países subtituladores tienen una mejor competencia en lenguas extranjeras que los países donde el doblaje es la práctica habitual (Talaván, 2013: 10).

La experiencia docente de los autores de este artículo sugiere que los alumnos de Grado de Educación_Primaria, no se sienten preparados lingüísticamente para impartir clases en inglés. Por ello, resulta imprescindible analizar la percepción de los docentes en formación sobre la calidad de la enseñanza en inglés en la universidad y valorar en qué medida este factor influye en el desarrollo de su autoeficacia docente como maestros de L2.

\section{Creencias de los futuros docentes}

Borg (2003) indica que el pensamiento docente tiene relación directa con las actuaciones de los maestros en el aula. Richards y Lockhart (1996) añaden que los maestros deben reflexionar sobre sus acciones y analizar las razones por las que las llevan a cabo. Los estudios realizados, manifiestan que los futuros docentes poseen un sistema inconsciente de creencias que, en ocasiones, difieren de los procedimientos sobre la enseñanza considerados apropiados (Barrios Espinosa, 2004, 2006 y 2014; Peacock, 2001). Estas creencias provienen de sus experiencias como alumnos (Pajares, 1992), de las observaciones que realizan durante las prácticas (Borg, 2003), de sus primeras experiencias docentes (Popko, 2005), de sus conocimientos metodológicos (Richards \& Lockhart, 1996) y de las conversaciones con otros colegas (Sengupta \& Xiao, 2002), que actúan como filtro ante los nuevos aprendizajes (Farrell, 2006; Pérez-Peitx \& Fons-Esteve, 2015). Dentro del contexto de la L1, Cambra y Palou (2007) consideran necesario partir de la reflexión sobre la propia experiencia para que se produzca una evolución en la acción docente del futuro maestro. Finalmente, Barrios (2014) concluye que los formadores deberían partir de estas ideas y adaptar los contenidos de los programas de estudios para ayudar a los maestros a reflexionar sobre la relación entre los nuevos conceptos y sus creencias preconcebidas.

\section{Autoeficacia docente}

En este ámbito, destaca el constructo de autoeficacia dentro de la teoría social cognitiva (Bandura, 1997, p. 3), definido como la opinión que cada persona tiene de su propia capacidad para desarrollar una acción y conseguir el resultado esperado (Prieto Navarro, 2007; Reoyo, 2013), en definitiva, su propia efectividad (Eslami y Fatahi, 2008). Wyatt (2010) define autoeficacia docente como las creencias en las capacidades de los docentes para 
promover el aprendizaje en las tareas y en los contextos cognitivo, meta-cognitivo, afectivo y social. Prieto (2007) va más allá al considerar que la opinión que cada profesor tiene de su capacidad docente dependerá del concepto que ellos mismos tengan de sus propias competencias.

La autoeficacia se caracteriza por estos elementos fundamentales: 1) es dinámica puesto que las experiencias vividas ayudan a que ésta aumente o disminuya (Blanco, 2009; Tschannen-Moran \& McMaster, 2009); 2) es cíclica porque los individuos no solo viven experiencias sino que también se benefician de ellas (Malinen, Savolainen \& Xu, 2012); 3) es multidimensional, ya que engloba procesos psicológicos cognitivos, motivacionales, afectivos y selectivos (Zimmerman, 2000); 4) es específica, porque no se puede hablar de poseer alta autoeficacia en todos los ámbitos y contextos (Knoblauch \& Woolfolk-Hoy, 2008).

Algunos estudios muestran la existencia de una correlación positiva entre la autoeficacia y aspectos como los logros académicos de los alumnos y su motivación (Gibson \& Dembo, 1984), las estrategias de control de los estudiantes, el liderazgo docente y la utilización de una metodología innovadora (Woolfolk \& Hoy, 1990), la implementación de la metodología existente (Ghaith \& Yaghi, 1997; Wertheim \& Leyser, 2002), la importancia que los profesores dan a la formación continua (Cousins \& Walker, 2000), la relación entre autoeficacia docente y resultados académicos (de la Torre \& Casanova, 2007), o el trabajo en equipo (Wyatt, 2010).

Dentro de los estudios de autoeficacia docente realizados en nuestro país, destaca la investigación de Prieto (2007), quien con su escala de medición de autoeficacia del profesor universitario invita a estos docentes a reflexionar sobre su capacidad para utilizar distintas estrategias didácticas. Con este análisis se obtiene información que permite relacionar la autoeficacia docente con sus estrategias pedagógicas y cómo esta relación afecta al proceso de enseñanza/aprendizaje. En esta línea, de la Torre y Casanova (2007) realizaron una comparativa entre la autoeficacia de docentes en formación y aquellos en ejercicio. Los primeros confían en su capacidad para afrontar las dificultades derivadas de los contextos familiares negativos de sus estudiantes, aspecto relacionado con la visión idealista y optimista que los futuros docentes tienen sobre la enseñanza (Woolfolk \& Hoy, 1990). Los segundos muestran una actitud más positiva sobre su acción docente y una mayor confianza en su capacidad de control del aula, aspecto que se fundamenta en las experiencias directas vivencias de éxito o fracaso- que constituyen una de las fuentes de la autoeficacia (Bandura, 1997). 
A nivel internacional, son escasas las investigaciones sobre autoeficacia docente en profesores no nativos que enseñen inglés como L2 en enseñanza reglada, probablemente debido al hecho de que la autoeficacia es un concepto complejo y difícilmente cuantificable (Prieto, 2007; Reoyo, 2013). Un estudio destacable dentro de las enseñanzas medias, es el realizado a 100 profesores venezolanos por Chacón (2005), cuyos resultados indican una relación positiva entre la autoeficacia y la competencia lingüística. Asimismo, Wyatt (2010) concluye que la autoeficacia docente del futuro maestro aumenta de distintas maneras según se relacione ésta con la enseñanza, con sus conocimientos sobre la enseñanza, con el currículum, con los enfoques metodológicos o con el contexto escolar.

\section{Formación de docentes de inglés}

En el Marco Europeo de Referencia para las Lenguas (MCERL) se enfatiza el papel de la competencia comunicativa y el aprendizaje de lenguas extranjeras desde edades tempranas, aspecto de relevancia para este estudio ya que se analiza la autoeficacia en la competencia lingüística de los futuros docentes en Primaria. A raíz de este documento, se han establecido nuevas demandas dentro de la formación de docentes de L2 entre las que destacan que la competencia lingüística de los docentes les permita utilizar el inglés como instrumento de comunicación en el aula. Sin embargo, algunos autores (Amengual-Pizarro, 2007, 2013; Barrios, 2006) sugieren que los programas de formación de maestros no prestan suficiente atención a su formación lingüística: según Barrios (2006), estos programas no dotan al futuro docente de un dominio de la L2 que les permita afrontar de manera fluida y correcta las situaciones cotidianas del aula. En esta línea, Amengual-Pizarro (2013) comenta que los actuales planes de estudio favorecen una formación más generalista, no siendo la formación lingüística actual suficiente para responder a las necesidades comunicativas que surgen en el aula de inglés. Amengual-Pizarro (2013) mantiene que las asignaturas de la especialidad se han reducido y los requisitos lingüísticos son menos exigentes ya que el inglés se considera una parte integral de la preparación del profesor generalista. Braine (citado en Choi y Lee, 2016) destaca el deseo de los futuros docentes por mejorar su competencia lingüística en inglés ya que la consideran una característica esencial en su formación.

\section{Objetivo}

Este artículo analiza la percepción sobre la formación lingüística recibida por un grupo de futuros maestros en los estudios de Grado de Maestro en Educación Primaria, mención inglés. El trabajo pretende explorar la autoeficacia docente de los estudiantes tras la realización de 
sus prácticas docentes para determinar posibles carencias. La investigación puede contribuir a un mejor entendimiento de la autoeficacia docente de los maestros al analizar -en la muestra seleccionada- su autoeficacia lingüística.

\section{Método}

Este trabajo sigue una metodología cualitativa y no pretende ser generalizable sino contribuir a un mejor entendimiento sobre el pensamiento de los docentes. Se realizó una entrevista estructurada a cada participante, tras la realización de sus prácticas docentes en $4^{\circ}$ del Grado de Educación Primaria, ya que en este momento han finalizado su instrucción formal y tienen la oportunidad de poner en práctica los conocimientos adquiridos.

Los datos fueron analizados utilizando la técnica del análisis del discurso en interacción en el que, partiendo de la palabra, el entrevistador se acerca cualitativamente al entrevistado analizando lo que dice y cómo lo dice (Palou, 2008, p. 146). Se siguió el modelo de Kerbrat-Orecchioni (2005) que incluye tres dimensiones: interlocutiva (mecánica de la entrevista: espacio y turnos de interacción), temática (posicionamiento y tipo de contribuciones, cambios o permanencia de tema), y declarativa (recursos discursivotextuales). Esta investigación se centrará en las dimensiones temática y declarativa (omitiendo la interlocutiva, en la que se sigue el orden natural de intervenciones entrevistador-pregunta y entrevistado-respuesta). La entrevista se divide en dos secuencias:

1) Se profundiza en aspectos personales y las razones que han llevado a los entrevistados a escoger estos estudios.

2) Se centra en la formación lingüística, la autoeficacia docente y las creencias sobre la enseñanza del inglés.

\section{Participantes}

En el curso 2014/2015 había 28 alumnos matriculados en el Practicum del $4^{\circ}$ del Grado de Educación Primaria. Se realizó una prueba para delimitar la competencia lingüística de los estudiantes en inglés y se agrupó a los alumnos en los 4 niveles resultantes según el MCERL: A2, B1, B2 y C1. Tras esta prueba, se seleccionó de manera aleatoria a 4 participantes (uno por nivel): 3 mujeres y 1 hombre, con edades entre los 21 y los 23 años. Los datos de los participantes se muestran en la Tabla 1: 


\begin{tabular}{|c|c|c|c|}
\hline Participante & Edad & Conocimientos del idioma & Razones para escoger la titulación \\
\hline Álvaro & 23 & nivel A2 & Mayores posibilidades laborales \\
\hline Natalia & 21 & nivel B1 & $\begin{array}{c}\text { Mención que más le atraía } \\
\text { Mayores posibilidades laborales }\end{array}$ \\
\hline María & 21 & nivel B2 & $\begin{array}{c}\text { Vocación profesional } \\
\text { Le gusta el inglés }\end{array}$ \\
\hline Noemí & 21 & nivel C1 & $\begin{array}{c}\text { Le gustan los niños } \\
\text { Le gusta el inglés }\end{array}$ \\
\hline
\end{tabular}

Tabla 1. Datos de los participantes

\section{Instrumento y procedimiento}

Se realizaron entrevistas estructuradas basada en un guion de preguntas cerradas que permitieron realizar comparaciones entre las respuestas de los entrevistados. Realizada de manera individual, grabada y transcrita en mayo de 2015, la entrevista tuvo un tiempo de duración de 15 minutos ya que al tratarse de preguntas cerradas los participantes se ciñeron a ellas.

La entrevista fue elaborada basándonos en las pautas de Rodríguez Gómez, Gil Flores y García Jiménez (1996) y Ruiz Olabuénaga (1999): se trata de una búsqueda de información y de la creación de un clima de confianza entre los interlocutores. La entrevista fue validada por 5 profesores ( 3 de primaria y 2 de universidad); se realizó una prueba piloto con tres alumnos de similares características a los de esta muestra para comprobar su idoneidad. Las preguntas se englobaron en dos bloques: 1) "Información biográfica" que recoge sus datos personales: sexo, edad, nivel de inglés, razones por las que escogieron estos estudios; 2) "El futuro docente y la competencia lingüístico-comunicativa", recoge la percepción sobre la formación lingüística recibida y su percepción de su competencia lingüística.

\section{Análisis de los datos}

A continuación, se presenta de manera resumida la información aportada para cada entrevistado, para posteriormente proceder a su análisis: 


\begin{tabular}{l|l|l|} 
Álvaro: & \\
$\begin{array}{l}\text { Competencia } \\
\text { lingüística }\end{array}$ & $\begin{array}{l}\text { Mi nivel de inglés, necesito mejorar bastante la expresión oral. } \\
\text { Mejoré muchísimo en vocabulario, escritura, también tengo que } \\
\text { mejorar la comprensión. }\end{array}$ \\
\hline Formación lingüística & Yo, la verdad es que aprendí bastante, sobre todo este año. \\
\hline $\begin{array}{l}\text { Preparación } \\
\text { lingüística ante las } \\
\text { intervenciones }\end{array}$ & $\begin{array}{l}\text { Me centraba más en la gramática porque era en lo que más } \\
\text { atrasado estaba en inglés. También es verdad que es muy } \\
\text { importante para mí adquirir más vocabulario sobre diferentes } \\
\text { contextos porque te ayuda a la hora de expresarte oralmente, } \\
\text { cuanto más conocimiento de vocabulario tengas más } \\
\text { posibilidades tienes a la hora de expresarte. }\end{array}$ \\
\hline $\begin{array}{l}\text { Enseñanza } \\
\text { aprendizaje de los } \\
\text { componentes de la } \\
\text { competencia }\end{array}$ & $\begin{array}{l}\text { Tienes que enseñar las cuatro destrezas y utilizar las diferentes } \\
\text { metodologías. } \\
\text { lingüística }\end{array}$ & $\begin{array}{l}\text { No, ahora mismo no. Yo creo que tendría que estar un año o así } \\
\text { dando clase de inglés. }\end{array}$ \\
\hline Autoeficacia docente
\end{tabular}

Tabla 2. Síntesis entrevista Álvaro

Álvaro considera que su inglés está en proceso de evolución. Al valorarlo utiliza el adverbio todavía, indicando su deseo de mejora y su propia exigencia personal, necesito mejorar bastante y concreta, la expresión oral. Su competencia lingüística es una de sus preocupaciones, menciona la palabra mejorar en tres ocasiones, no se siente satisfecho con ella y desea que prospere. Nota que ha progresado, pero no lo suficiente en su comprensión oral.

En cuanto a la formación recibida, personaliza y enfatiza su respuesta positiva Yo la verdad es que aprendí bastante. Se siente realmente satisfecho con lo aprendido, sin explicitar los aspectos.

Álvaro prepara con detenimiento sus intervenciones, especialmente la gramática: porque era en lo que más atrasado estaba en inglés y el vocabulario: cuanto más conocimiento de vocabulario tengas, más posibilidades tienes a la hora de expresarte, por ser también sus propias flaquezas.

Aunque otorga importancia a las cuatro destrezas, destaca como fundamental la gramática y el vocabulario, indicando así que los conocimientos lingüísticos deben ser 
simultáneos a los metodológicos: utilizar las diferentes metodologías para adaptarte a los distintos alumnos.

Álvaro manifiesta no estar preparado para enseñar No, ahora mismo no. Explica que esa seguridad la adquirirá con el tiempo: yo creo que tendría que estar un año o así dando clase de inglés. Además, comenta tener dificultades para expresarse y comprender y cree que, en ocasiones, los alumnos no le comprenden. Por estas dificultades, se puede decir que posee una autoeficacia docente negativa.

Natalia:

\begin{tabular}{|c|c|}
\hline $\begin{array}{l}\text { Competencia } \\
\text { lingüística }\end{array}$ & $\begin{array}{l}\text { Mi nivel de inglés es bueno de forma escrita, en lectura y escucha } \\
\text { está bien. La fluidez oral no la tengo desarrollada todavía y } \\
\text { debería mejorarla. }\end{array}$ \\
\hline on lingüística & $\begin{array}{l}\text { Yo creo que es muy difícil que en el tiempo que se le dedica a la } \\
\text { asignatura llegues al nivel que se tiene que tener. Yo creo que } \\
\text { tienes que tener un nivel tú ya previo y estudiar fuera inglés. } \\
\text { Debería centrarse en enseñarnos un lenguaje más técnico. }\end{array}$ \\
\hline $\begin{array}{l}\text { Preparación } \\
\text { lingüística ante las } \\
\text { intervenciones }\end{array}$ & $\begin{array}{l}\text { Me planteo la serie de preguntas que quiero hacerles previamente } \\
\text { para que estén gramaticalmente correctas, las leo y pronuncio en } \\
\text { casa para acordarme de ellas, aunque luego llegues a clase, no las } \\
\text { digas, pero las tengo siempre apuntadas por si acaso me quedo } \\
\text { en blanco porque siempre si hay una palabra que no te sale } \\
\text { puedes mirar el papel, nadie se da cuenta de que realmente que } \\
\text { no te sale la palabra. }\end{array}$ \\
\hline $\begin{array}{llr}\text { Enseñanza } & & \text { y } \\
\text { aprendizaje de los } & \\
\text { componentes de la } \\
\text { competencia } & & \\
\text { lingüística } & & \end{array}$ & $\begin{array}{l}\text { Me he dado cuenta que es importante que lo sepan hablar, pero a } \\
\text { la vez que también sepan escribirlo. }\end{array}$ \\
\hline Autoeficacia docente & $\begin{array}{l}\text { Yo no me veo preparada para enseñar inglés. No tengo la fluidez } \\
\text { oral para dar la clase con soltura o la correcta pronunciación con } \\
\text { palabras nuevas, para que ellos las aprendan. }\end{array}$ \\
\hline
\end{tabular}

Tabla 3. Síntesis entrevista Natalia

Natalia opina sobre su nivel de inglés yo creo que es bueno y especifica de forma escrita, en lectura y escucha está bien. Considera que sus habilidades escritas y su comprensión oral son buenas. Luego matiza: la fluidez oral no la tengo desarrollada todavía, muestra entonces descontento, con el uso del adverbio todavía se percibe que es hasta este momento y desea que la situación cambie debería mejorarla.

Cree que el nivel deseable debería ser superior al que se llega con la formación recibida: creo que es muy dificil que en el tiempo que se le dedica a la asignatura llegues al nivel que se tiene que tener. Continúa, enfatizando su respuesta desde la primera persona del 
singular: yo creo que, y con sentido de obligatoriedad tienes que tener, que, para indicarla, se sitúa en la segunda persona del singular y aclara un nivel tú ya previo. Explica que se debe acceder con unos conocimientos, a los que se añaden estancias de estudio del idioma estudiar fuera inglés. Y suma su deseo de una formación más técnica debería enseñarnos un lenguaje más técnico, apreciación que está en la línea de los estudios realizados por Amengual-Pizarro (2013) y Barrios (2006).

En cuanto a su preparación, me planteo la serie de preguntas que quiero hacerles. Luego, se corrige a sí misma las pongo para que estén gramaticalmente correctas. Después, las practica las leo y pronuncio en casa para acordarme de ellas. Natalia se esfuerza mucho, utiliza el reflexivo y realiza una descripción detallada me planteo, las pongo, las leo y pronuncio. A continuación, con la conjunción adversativa plantea lo que ocurre realmente en el aula, aunque luego llegues a clase y no las digas. Sin embargo, continúa, pero las tengo siempre apuntadas por si acaso me quedo en blanco, hecho que agradece. Es entonces cuando explica si hay una palabra que no te sale puedes mirar el papel. Es un alivio el pensar que su desconocimiento no será notado por los alumnos nadie se da cuenta de que realmente no te sale la palabra.

Natalia posee una autoeficacia docente negativa por sus comentarios en relación a su competencia lingüística, que no considera apropiada, y comenta Yo no me veo preparada. Cree no estar capacita para ejercer y explica: no tengo la fluidez oral para dar la clase con soltura o la correcta pronunciación con palabras nuevas, afirmaciones basadas en su limitada competencia lingüística, especialmente su habilidad oral, fundamental para un docente de inglés.

María: 


\begin{tabular}{l|l|}
\hline $\begin{array}{l}\text { Competencia } \\
\text { lingǘstica }\end{array}$ & $\begin{array}{l}\text { Bien, alto, quizás no tan alto como me gustaría pero sí que me } \\
\text { puedo defender con el idioma. Y un aspecto que me encantaría } \\
\text { mejorar sería el, para ser un buen profesor, sería la expresión } \\
\text { oral. }\end{array}$ \\
\hline $\begin{array}{l}\text { Formación lingüística } \\
\text { Preparación }\end{array}$ & Están bien \\
$\begin{array}{l}\text { lingüística ante las } \\
\text { intervenciones }\end{array}$ & Le dedicaba bastante tiempo. \\
\hline $\begin{array}{l}\text { Enseñanza de los } \\
\text { aprendizaje desarrollar las cuatro skills. } \\
\text { componentes de la } \\
\text { competencia }\end{array}$ & \\
lingüística &
\end{tabular}

Tabla 4. Síntesis entrevista María

María opina sobre su nivel de inglés (B2) bien, alto, quizás no tan alto como me gustaría. Tiene una alta percepción, aunque siente que debería mejorar su expresión oral, y una opinión positiva hacia la formación lingüística recibida en inglés.

En cuanto a la preparación de las clases, dedica tiempo a los aspectos lingüísticos especialmente. Explica no me gustaba tener dudas, entonces pensaba todo muy bien y detalla qué pregunta podía hacer, cómo sería la estructura de esa pregunta. Luego, añade sí que trataba de escribir cómo serían las preguntas, bien formuladas y en cuanto al vocabulario y la gramática. La pronunciación es otro aspecto que le preocupa y en el que no se permite vacilar, dice yo no puedo dudar.

María destaca los aspectos más importantes a enseñar y dice yo creo... que sean capaces de desarrollar las cuatro skills. Ve importante crear oportunidades para utilizar el idioma en ejercicios de temática interesante y significativa para los niños.

María tiene una autoeficacia docente positiva debido a que su propia valoración sobre su competencia lingüística también lo es. 
Noemi:

\begin{tabular}{|c|c|}
\hline $\begin{array}{l}\text { Competencia } \\
\text { lingüística }\end{array}$ & $\begin{array}{l}\text { Bueno, de mi nivel de inglés yo no lo adquirí en la universidad. } \\
\text { La Universidad no me dio nada, a pesar de que siempre hay cosas } \\
\text { que aprendes, detalles. Pero lo que es mi nivel de inglés, si lo } \\
\text { tomamos todo, viene de atrás. }\end{array}$ \\
\hline Formación lingüística & $\begin{array}{l}\text { Yo creo que lo de la Universidad sí que está bien montado; pero } \\
\text { ¿qué pasa?, antes era una diplomatura de tres años enteros la } \\
\text { especialidad de inglés, no se puede comparar con lo que se hacía } \\
\text { antes... pero el problema no está en la asignatura sino en el } \\
\text { tiempo que se le dedica a la asignatura. }\end{array}$ \\
\hline $\begin{array}{l}\text { Preparación } \\
\text { lingüística ante las } \\
\text { intervenciones }\end{array}$ & $\begin{array}{l}\text { Yo de construcción o de improvisar sobre la marcha a la hora de } \\
\text { hablar no tengo un gran problema. Hay determinadas cosas que } \\
\text { hay que revisar antes de enseñarlo a los niños porque no se puede } \\
\text { improvisar. }\end{array}$ \\
\hline $\begin{array}{llr}\text { Enseñanza } & & \text { y } \\
\text { aprendizaje de } & \text { los } \\
\text { componentes } & \text { de } & \text { la } \\
\text { competencia } & & \\
\text { lingüística } & \end{array}$ & Es la comunicación oral \\
\hline Autoeficacia docente & $\begin{array}{l}\text { Por un lado sí, por otro no. Depende de la metodología que vaya } \\
\text { a utilizar, con Jolly Phonics y CLIL (Content and Language } \\
\text { Integrated Learning) no me veo muy capaz, pero si es buscar } \\
\text { ejercicios o utilizar el libro, sí. }\end{array}$ \\
\hline
\end{tabular}

Tabla 4. Síntesis entrevista Noemí

Noemí manifiesta tener un nivel de inglés elevado tras muchos años de estudio, previos a comenzar el Grado, mi nivel de inglés yo no lo adquirí en la universidad. Y sentencia: la universidad no me dio nada. Y luego, a través de la locución preposicional a pesar de rectifica siempre hay cosas que aprendes, con el adverbio siempre se muestra un carácter positivo y absoluto y termina la oración detalles, indicando que lo aprendido fue insignificante.

Se observa un dilema, comienza de manera positiva Yo creo que lo de la universidad sí que está bien montado. Y se pregunta, pero ¿qué pasa? Entonces explica las diferencias entre el plan de estudios de la Diplomatura y el actual. Establece una relación entre el antes no se puede comparar con lo que se hacía antes y el de ahora, el actual no le satisface por la cantidad de tiempo dedicada a la instrucción. Termina aclarando el problema no está en la asignatura sino en el tiempo que se le dedica a la asignatura. 
Las prácticas escolares le han servido para afianzar su creencia en que el aspecto oral es el más importante y hay que potenciarlo. La interacción es un aspecto a destacar, está directamente relacionada con su seguridad y alta competencia en la L2. Quizás por esta razón, no hace ningún comentario sobre su preparación lingüística, su nivel de inglés le permite improvisar y no sentirse nerviosa ante las dudas que pudieran surgir en el aula.

Ante la pregunta de si se siente preparada para enseñar, se aprecia un dilema: Por un lado sí, por otro no. Ante las nuevas metodologías: Jolly Phonics y CLIL dice insegura no me veo muy capaz. Sin embargo, sí lo está, si se trata de buscar ejercicios y dice hay que buscar o de usar el libro.

En cuanto a su autoeficacia docente, Noemí no considera que esté preparada para enseñar, debido fundamentalmente a aspectos metodológicos, no por su competencia lingüística. Como aquí solo valoramos esta última, se puede decir que Noemí tiene una autoeficacia docente positiva.

\section{Discusión de resultados}

Los participantes destacan la importancia de alcanzar, antes de finalizar sus estudios universitarios, una competencia lingüística en inglés que les permita expresarse de manera correcta y fluida. Los alumnos deben alcanzar un B2 según la Guía Docente de "Inglés: Destrezas Comunicativas", aunque este nivel no resulta suficiente para los encuestados. Esta aseveración está en consonancia con estudios realizados en este sentido (Amengual-Pizarro, 2007 \& 2013; Barrios, 2006; Berry, 1990; Pawlak, 2011). Berry (1990) establece que una mayor confianza en la competencia lingüística por parte de los maestros facilitaría un mayor uso de la L2 en el aula y su percepción sobre su competencia docente aumentaría. Esta afirmación es clara en participantes como María (B2) y Noemí (C1), con una competencia lingüística intermedia-alta.

Los entrevistados con un nivel inferior al B2 tienen una baja percepción de su autoeficacia docente, lo que achacan a su menor competencia lingüística, especialmente su expresión oral, que desean mejorar. Comentan que esta carencia está en consonancia con la pobre formación oral recibida en sus clases de L2 en Primaria. Por ello, reclaman una formación más extensa en la que se potencien las estrategias comunicativas que consideran fundamentales.

Este es un aspecto en el que merece la pena detenerse: los entrevistados recordaron la instrucción recibida en la asignatura de inglés durante sus estudios de Educación Primaria 
como una formación basada en la enseñanza de la gramática y el vocabulario, en la que el libro de texto era el recurso fundamental y la L2 tenía poco protagonismo. La participante con nivel C1 incluso manifestó que su alta competencia lingüística no se debía a la formación recibida en la escuela, sino que la había adquirido en otros centros. Con el fin de paliar esta deficiente competencia oral, los participantes proponen una enseñanza de la L2 basada en clases dinámicas en las que prime la interacción entre alumno-alumno y profesor-alumno, a través de la utilización de una variedad de recursos y en la que destaque el aspecto lúdico. Esta percepción está en consonancia con la formación que reciben en el Grado, pero, de acuerdo a sus manifestaciones, difiere de la recibida durante su etapa en Primaria. La utilización de recursos que fomenten la comunicación hará que el discente se sienta motivado por lo que su aprendizaje y participación se verán favorecidos.

Los participantes dicen preparar minuciosamente sus intervenciones desde el punto de vista lingüístico, especialmente en aspectos como vocabulario, gramática y pronunciación, para evitar errores y anticiparse a aquellas dudas que pudieran ser planteadas más allá del contenido estricto de las clases. Además, los testimonios obtenidos confirman la necesidad de una formación lingüística específica de la profesión docente que ayude a los maestros a incorporar un vocabulario adecuado, que ayude a sus comunicaciones con el alumnado y que evite errores en los mensajes, además de reduplicaciones en las explicaciones, aspectos ya tratados por Barrios (2006) y Amengual-Pizarro (2013) y que quedan de manifiesto en los nuevos Grados.

Según los resultados del estudio, se aprecia que la autoeficacia docente de los participantes difiere de manera clara dependiendo de su competencia lingüística. Mientras que aquellos participantes con un B2 o superior se sienten capacitados para enseñar, los que poseen un nivel inferior se muestran inseguros. Por esta razón, reclaman una mayor formación lingüística que les proporcionaría una mayor confianza.

\section{Reflexiones finales}

La creencia de autoeficacia docente indica el grado en el que los maestros se consideran capaces de planificar y desarrollar acciones con el fin de conseguir unos resultados entre sus alumnos. El interés de esta investigación se centra en mostrar la opinión de los futuros docentes sobre su formación lingüística y su autoeficacia docente, constructo relevante en la formación del profesorado. 
Los participantes cuyos niveles de inglés son inferiores al nivel B2 perciben que su competencia lingüística en la L2 no es suficiente. Para superar estas carencias, reclaman una mayor formación en lengua inglesa, concretamente en vocabulario específico de la profesión y en el tiempo dedicado a la asignatura. Por otro lado, los entrevistados conceden gran importancia al componente oral en la formación en L2 de los alumnos de Primaria, hecho relacionado con sus declaraciones sobre las pocas oportunidades que tuvieron de expresarse oralmente durante su etapa en Primaria.

Además, estos participantes tienen una percepción baja de su autoeficacia docente, no sintiéndose preparados para enseñar porque consideran que necesitan mejorar su competencia lingüística en aspectos orales, principalmente la fluidez. Sin embargo, los entrevistados cuyo nivel es superior al nivel B2, se sienten preparados desde un punto de vista lingüístico, siendo su autoeficacia docente percibida positiva.

Como sucede en toda investigación, el presente estudio presenta algunas limitaciones: se trata de un estudio exploratorio basado en una muestra de 4 participantes. Además, la investigación se basa en una única entrevista realizada tras las prácticas docentes de los participantes, por lo que no se ha podido realizar una comparativa entre sus opiniones antes de la formación específica en inglés que ofrece la mención y después. La investigación en la autoeficacia docente de los maestros de inglés puede tener implicaciones metodológicas en el ámbito de la formación del profesorado (tanto para los profesores encargados de impartir materias sobre metodología como para los que enseñan lengua instrumental en los estudios de grado), así como en los propios planes de estudio de las titulaciones de magisterio, que debieran prestar más atención a este constructo. Asimismo, resulta necesario que se realicen más investigaciones en esta línea para lograr una mayor comprensión de la autoeficacia docente y poder mejorar la formación de los maestros en nuestro país. Este trabajo puede ser el punto de partida para futuras investigaciones de carácter longitudinal para que, como apunta, Wyatt (2015), se pueda explorar más profunda y ampliamente en sus creencias de autoeficacia. También sería interesante realizar investigaciones que analizaran las relaciones existentes entre la autoeficacia lingüística y metodológica en los docentes de inglés en Educación Primaria. Igualmente, es necesario investigar la autoeficacia docente y las percepciones de los futuros maestros de inglés mediante otros enfoques y aproximaciones que utilicen diseños de investigación cuantitativa y mixta, para poder establecer correlaciones entre ambas y medir el impacto que tienen determinadas variables -como el nivel de ingléssobre el desarrollo de la autoeficacia docente. 


\section{Referencias}

Amengual-Pizarro, M. (2007). How to respond to the demands set by the communicative approach? New challenges L2 teachers face in the classroom. The European Journal of Teacher Education, 30(1), 63-73. Doi: http://dx.doi.org/10.1080/02619760601120007.

Amengual-Pizarro, M. (2013). Primary education degrees in Spain: do they fulfill the linguistic and pedagogic needs of future teachers? Vigo International Journal of Applied Linguisitics, 10, 9-27. Recuperado de http://webs.uvigo.es/vialjournal/pdf/Vial-2013Article1.pdf.

Bandura, A. (1997). Self-efficacy. The exercise of control. Englewood Cliffs, NJ: Prentice Hall.

Barrios Espinosa, M. E. (2004). El contraste entre la planificación de las clases y la enseñanza interactiva en la intervención docente autónoma de futuros docentes de inglés durante las Prácticas de Enseñanza. Porta Linguarum, 2, 31-55. Recuperado de http://www.ugr.es/ portalin/articulos/PL numero22/6\%20\%20ELVIRA\%20BARRIOS. pdf.

Barrios Espinosa, M. E. (2006). Dificultades y carencias que experimentan y evidencian futuros docentes de inglés durante su intervención docente autónoma en las prácticas de enseñanza. REIFOP, 9(1), 1-12.

Barrios Espinosa, M. E. (2014). Creencias sobre el aprendizaje de una lengua del futuro profesorado de inglés de Educación Primaria. Porta Linguarum, 22, 73-93. Recuperado de

http://www.ugr.es/ portalin/articulos/PL numero22/6\%20\%20ELVIRA\%20BARRIOS. pdf.

Barcelos, A. M. F. (2003). Researching beliefs about SLA: A critical review. En P. Kalaja \& A.M.F. Ferreira Barcelos (eds.), Beliefs about second language acquisition. New research approaches (pp. 7-33). Países Bajos: Kluwer Academic Publishers.

Berry, R. (1990). The role of language improvement in in-service training: Killing two birds with one stone. System, 18(1), 97-105. Doi: http://dx.doi.org/10.1016/0346251X(90)90032-Z.

Birello, M. (2012). Teacher cognition and language teacher education: beliefs and practice. A conversation with Simon Borg. Bellaterra Journal of Teaching \& Learning Language \& Literature, 5 (2), 88-94. Doi: http://dx.doi.org/10.5565/rev/jt13.464.

Blanco, Á. (2009). El modelo cognitivo social del desarrollo de la carrera: revisión de más de una década de investigación empírica. Revista de Educación, 350, 423-445. Recuperado de: http://www.revistaeducacion.mec.es/re350/re350 18.pdf.

Borg, S. (2003). Teacher cognition in language teaching: A review of research on what language teachers think, know, believe, and do. Language Teaching, 36(2), 81-109. Doi: $10.1017 / \mathrm{S} 02614444803001903$.

Borg, S. (2009). Introducing language teacher cognition. Recuperado de http://www.education.leeds.ac.uk/research/files/145.pdf.

Cambra, M., y Palou, J. (2007). Creencias, representaciones y saberes de los profesores de lenguas en las nuevas situaciones plurilingües escolares de Cataluña. Cultura y Educación, 19(2), 149-163.

Chacón, C. T. (2005). Teachers' perceived efficacy among English as a foreign language teachers in middle schools in Venezuela. Teaching and Teacher Education, 21, 257272. Doi: http://dx.doi.org/10.1016/j.tate.2005.01.001.

Choi, E., y Lee, J. (2016). Investigating the relationship of target language proficiency and self-efficacy among nonnative EFL teachers. System, 58, 49-63. http://dx.doi.org/10.1016/j.system.2016.02.010 
Consejo de Europa. (2002). Marco Común de Referencia Europeo. Versión española del Instituto Cervantes. Recuperado de http://cvc.cercantes.es/obref/marco.

Cousins, J., \& Walker, C. (2000). Predictors of educators' valuing of systemic inquiry in schools. Canadian Journal of Program Evaluation, num. extraordinario, 25-52.

Covarrubias, C., y Mendoza, M. (2013). La teoría de autoeficacia y el desempeño docente: el caso de Chile. Estudios Hemisféricos y Polares, 4 (2), 107-123.

de la Torre Cruz, M. J., \& Casanova Arias, P. F. (2007). Comparative analysis of experiences of efficacy in in-service and prospective teachers. Teaching and Teacher Education, 23(5), 641 - 652. Doi: http://dx.doi.org/10.1016/j.tate.2007.02.005.

Eslami, Z. R., y Fatahi, A. (2008). Teachers' Sense of Self-Efficacy, English Proficiency, and Instructional Strategies: A Study of Nonnative EFL Teachers in Iran. TESL-EJ, 11(4), 1-19. Recuperado de http://files.eric.ed.gov/fulltext/EJ898136.pdf

European Commission (2012a). Eurobarometre 386: Europeans and their languages. Recuperado de http://ec.europa.eu/public opinion/archives/ebs/ebs 386 en.pdf.

European Commission (2012b). European survey on language competences. Recuperado de http://ec.europa.eu/languages/library/studies/executive-summary-eslc en.pdf.

Eurydice. (2012). Key data on teaching languages at school. Recuperado de http://ec.europa.eu/languages/policy/strategic-framework/documents/key-data2012 en.pdf.

Farrell, T. S. C. (2006). The teacher is an octopus: uncovering pre-service English language teachers' prior beliefs through metaphor analysis. RELC Journal, 37(2), 236-248.

Ghaith, G., y Yaghi, H. (1997). Relationships among experience, teacher efficacy and attitudes toward the implementation of instructional innovation. Teaching and Teacher Education, 13(4), 451-458. Doi: http://dx.doi.org/10.1016/S0742-051X(96)00045-5.

Gibson, L. S., y Dembo, M. H. (1984). Teacher efficacy: a construct validation. Journal of Educational Psychology, 76, 569-582. Doi: http://dx.doi.org/10.1037/00220663.76.4.569.

Kerbrat-Orecchioni, C. (2005). Le discours en interaction. París: Armand Colin

Knoblauch, D., y Woolfolk-Hoy, A. (2008). Maybe I can teach those kids. The influence of contextual factors on student teachers' efficacy beliefs. Teaching and Teacher Education, 24(1), 166-179. Doi: http://dx.doi.org/10.1016/j.tate.2007.05.005.

Malinen, O. P., Savolainen, H., y Xu, J. (2012). Beijing in-service teachers' self-efficacy and attitudes towards inclusive education. Teaching and Teacher Education, 28(4), 526-534. Doi: http://dx.doi.org/10.1016/j.tate.2011.12.004.

Mojavezi, A., y Tamiz, M. P. (2012). The impact of teacher self-efficacy on the students' motivation and achievement. Theory and Practice in Language Studies, 2(3), 483-491. Doi: http://dx.doi.org/10.4304/tpls.2.3.483-491.

Pajares, F. (1992). Teachers' beliefs and educational research: Cleaning up the messy construct. Review of Educational Research, 62(3), 247-265. Doi: http://dx.doi.org/10.3102/00346543062003307.

Palou, J. (2008). L'ensenyament i l'aprenentatge del català com a primera llengua a l'escola. Creences $i$ actuacions dels Mestres amb relació a les activitatss de llegngua oral a l'etapa primària. (Tesis doctoral). Barcelona: Institut d'Estudis Catalans.

Pawlak, M. (2011). The role of in-service training for language teachers in the domain of language competence. Glottodidactica. An International Journal of Applied Linguistics, 38, 21-30.

Peacock, M. (2001). Pre-service ESL teachers' beliefs about second language learning: a longitudinal study. System, 29(3), 177-195. Doi: http://dx.doi.org/10.1016/S0346$\underline{251 \times(01) 00010-0 .}$. 
Pendergast, D., Garvis, S., y Keogh, J. (2011). Pre-service student-teacher self-efficacy beliefs: An insight into the making of teachers. Australian Journal of Teacher Education, 36(12), 46-58. Doi: http://dx.doi.org/10.14221/ajte.2011v36n12.6.

Pérez-Peitx, M., y Fons-Esteve, M. (2015). Beliefs and tensions of kindergarten preservice students: a three case longitudinal study. Multidisciplinary journal for education, social and technological sciences, 2(1), 63-77. Doi: http://dx.doi.org/10.4995/muse.2015.2235.

Popko, J. (2005). How MA-TESOL students use knowledge about language in teaching ESL classes. Applied Linguistics and Language Teacher Education, 4, 387-403. Doi: http://dx.doi.org/10.1007/1-4020-2954-3 22.

Prieto Navarro, L. (2001). La autoeficacia en el contexto académico. Exploración bibliográfica comentada. Miscelánea Comillas, 59, 281-292.

Prieto Navarro, L. (2007). Autoeficacia del profesor universitario: eficacia percibida y práctica docente (vol. 15). Madrid: Narcea Ediciones.

Reoyo, N. (2013). Autoeficacia docente en Educación Secundaria Obligatoria: perspectivas de alumnos, profesores y futuros profesores. (Tesis doctoral inédita). Valladolid: Universidad de Valladolid.

Richards, J. C., y Lockhart, C. (1996). Reflective teaching in second language classrooms. Cambridge: Cambridge University Press.

Rodríguez Gómez, G., Gil Flores, J., y García Jiménez, E. (1996). Metodología de la investigación cualitativa. Málaga: Ediciones Aljibe.

Ruiz Olabuénaga, J. I. (1999). Metodología de la investigación cualitativa. Bilbao: Universidad de Deusto.

Rubio, F. D., y Martínez Lirola, M. (2008). La comunicación oral del inglés en España: Influencia de los aspectos educativos y mediáticos en el éxito del aprendizaje. Revista de Enseñanza Universitaria, 32, 51-63.

Sengupta, S., y Xiao, M. (2002). The contextual reshaping of beliefs about L2 writing: Three teachers' practical process of theory construction. TESL-EJ, 6(1)A1. Recuperado de http://www.tesl-ej.org/ej21/a1.html.

Talaván, N. (2013). Subtitulación en el aprendizaje de lenguas extranjeras. Barcelona: Octaedro.

Tschannen-Moran, M., y McMaster, P. (2009). Sources of self-efficacy: four professional development formats and their relationship to self-efficacy and implementation of a new teaching strategy. The Elementary School Journal, 110(2), 228-245. Doi: http://dx.doi.org/10.1086/605771.

Tschannen-Moran, M., y Woolfolk, A. (2007). The differential antecedents of self-efficacy beliefs of novice and experienced teachers. Teaching and Teacher Education, 23(6), 944-956. Doi: http://dx.doi.org/10.1016/j.tate.2006.05.003.

Wertheim, C., y Leyser, Y. (2002). Efficacy beliefs, background variables, and differentiated instruction of Israeli prospective teachers. The Journal of Educational Research, 96(1), 54-63. Doi: http://dx.doi.org/10.1080/00220670209598791.

Woolfolk, A., y Hoy, W. (1990). Prospective teachers' sense of efficacy and beliefs about control. Journal of Educational Psychology, 82, 81-91.

Wyatt, M. (2010). An English teacher's developing self-efficacy beliefs in using groupwork. System, 38, 603-613. Doi: http://dx.doi.org/10.1016/j.system.2010.09.012.

Wyatt, M. (2015). Using qualitative research methods to assess the degree of fit between teachers' reported self-efficacy beliefs and their practical knowledge during teacher education. Australian Journal of Teacher Education, 40(1), 117-145. Doi: http://dx.doi.org/10.14221/ajte.2015v40n1.7. 
Zimmerman, B. J. (2000). Self-efficacy: an essential motive to learn. Contemporary Educational Psychology, 25, 89-91. Recuperado de https://sydney.edu.au/educationportfolio/ei/news/pdfs/Zimmerman\%202000.pdf.

\section{Información sobre los autores:}

Ana Fernández-Viciana realiza su tesis doctoral en el área de Didáctica de la Lengua y la Literatura de la Universidad de Oviedo, donde trabaja como profesora en el centro adscrito Facultad Padre Ossó. Sus líneas de investigación son el pensamiento de los profesores, las creencias de autoeficacia y la formación del profesorado. Tiene previsto defender su tesis en la primavera de 2017.

Email: anafervic@facultadpadreosso.es

Alberto Fernández-Costales es profesor del Área de Didáctica de la Lengua y la Literatura de la Universidad de Oviedo. Sus principales líneas de investigación son la enseñanza del inglés como lengua extranjera, la educación bilingüe, el multilingüismo y el Aprendizaje Integrado de Contenidos y Lenguas Extranjeras (AICLE).

E-mail: fernandezcalberto@uniovi.es

Para citar este artículo:

Fernández-Viciana, A., \& Fernández-Costales, A. (2017). El pensamiento de los futuros maestros de inglés en Educación Primaria: creencias sobre su autoeficacia docente. Bellaterra Journal of Teaching \& Learning Language \& Literature, 10(1), 42-60. DOI: http://dx.doi.org/10.5565/rev/jt13.684 